\title{
Thermally Switchable Olefin Metathesis Initiators bearing Chelating Carbenes - Influence of the Chelates's Ring Size
}

\author{
Christian Slugovc, * Daniel Burtscher, Franz Stelzer, and Kurt Mereiter
}

Institute of Chemistry and Technology of Organic Materials (ICTOS), Graz University of Technology,

Stremayrgasse 16, A-8010 Graz, Austria, and

Institute of Chemical Technologies and Analytics, Vienna University of Technology,

Getreidemarkt 9/164, A-1060 Vienna, Austria

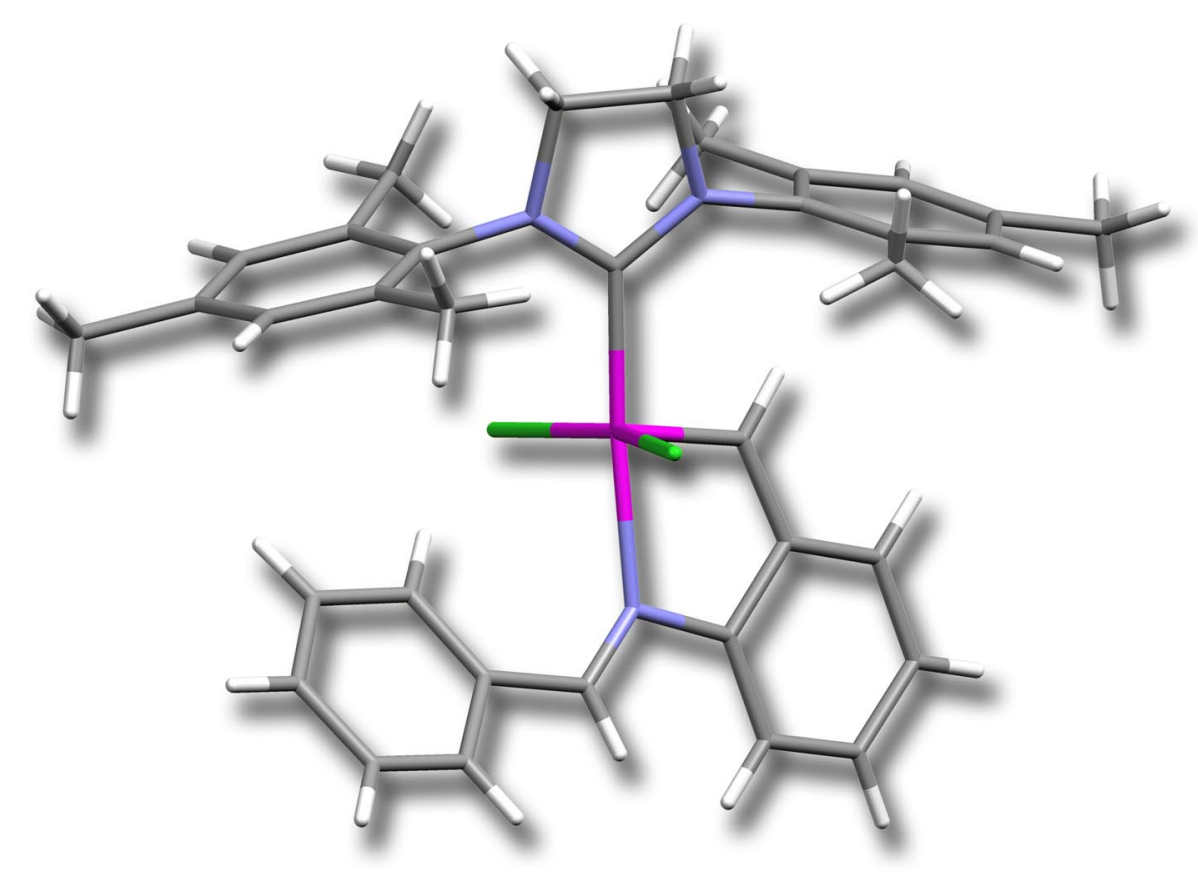




\section{Experimental Section}

General Comments. $\left(\mathrm{H}_{2} \mathrm{IMes}\right)$ (pyridine) ${ }_{2} \mathrm{Cl}_{2} \mathrm{Ru}=\mathrm{CHPh}(3)$, ( \pm )-endo,exo-bicyclo[2.2.1] hept-5-ene2,3-dicarboxylic acid diethyl ester (6), ${ }^{1}$ 2-vinylbenzaldehyde ${ }^{2}$ and 2-vinylphenylamine ${ }^{3}$ were prepared according to the literature. Benzaldehyde and aniline were purchased from Aldrich and were used as received. The weight-average molecular weights $\left(M_{W}\right)$ and polydispersity indices $(P D I)$ were determined by gel permeation chromatography using THF as the solvent in the following arrangement: Merck Hitachi L6000 pump, separation columns of Polymer Standards Service, 8 x 300 mm STV $5 \mu \mathrm{m}$ grade size $\left(10^{6} \AA, 10^{4} \AA\right.$, and $\left.10^{3} \AA\right)$, refractive index detector from Wyatt Technology, model Optilab DSP Interferometric Refractometer. Polystyrene standards purchased from Polymer Standard Service were used for calibration. ${ }^{1} \mathrm{H}-\mathrm{NMR}$ spectra were recorded on a Varian INOVA $500 \mathrm{MHz}$ spectrometer operating at $499.803 \mathrm{MHz}$ and were referenced to $\mathrm{SiMe}_{4}$, the relaxation delay was set to $10 \mathrm{~s} .{ }^{13} \mathrm{C}\left\{{ }^{1} \mathrm{H}\right\}$ NMR spectra were recorded on a Varian INOVA $500 \mathrm{MHz}$ spectrometer operating at $125.687 \mathrm{MHz}$ and were referenced to $\mathrm{SiMe}_{4}$. Differential scanning calorimetry (DSC) was performed on a Pyris Diamond DSC calibrated by using an indium standard.

\section{Synthesis of $(E)$-N-(2-Vinylbenzylidene)benzenamine (1)}

A mixture of aniline $(1.7 \mathrm{~g}, 1.8 \mathrm{mmol})$ and $\mathrm{NEt}_{3}(0.84 \mathrm{~mL})$ in $25 \mathrm{~mL} \mathrm{CH}_{2} \mathrm{Cl}_{2}$ was cooled to $-50^{\circ} \mathrm{C}$. Subsequently $\mathrm{TiCl}_{4}(80 \mu \mathrm{L})$ dissolved in $2 \mathrm{~mL} \mathrm{CH}_{2} \mathrm{Cl}_{2}$ was added slowly under an inert atmosphere of argon followed by slow addition of 2-vinylbenzaldehyde $(210 \mathrm{mg}, 1.6 \mathrm{mmol})$. The cooling bath was removed and the reaction was stirred for $20 \mathrm{~h}$ at room temperature. After removal of the solvent the resulting residue was crushed with a spatula. After addition of $10 \mathrm{mLEt}_{2} \mathrm{O}$ the resulting suspension was stirred vigorously until the solid content was grounded to a fine powder. This powder was sucked off and evaporation of the ether released a yellow oil, which solidified upon addition of pentane. The product was recrystallized from pentane. Yield: $56 \mathrm{mg}(17 \%)$

Anal. Calcd for $\mathrm{C}_{15} \mathrm{H}_{13} \mathrm{~N}$ (MW: 207.27): C, 86.92; H, 6.32. Found: C, 86.73; H, 6.44.

${ }^{1} \mathrm{H}-\mathrm{NMR}\left(\delta, 20^{\circ} \mathrm{C}, \mathrm{CDCl}_{3}, 500 \mathrm{MHz}\right): 8.82(\mathrm{~s}, 1 \mathrm{H}, \mathrm{N}=\mathrm{CH}), 8.12\left(\mathrm{~d}, 1 \mathrm{H}, \mathrm{Ph}^{6}\right), 7.54\left(\mathrm{~d}, 1 \mathrm{H}, \mathrm{Ph}^{3}\right)$,

$7.47\left(\mathrm{t},{ }^{3} J_{H H}=7.3 \mathrm{~Hz} 1 \mathrm{H}, \mathrm{Ph}^{4}\right), 7.43-7.40\left(\mathrm{~m}, 3 \mathrm{H}, \mathrm{Ph}^{5,3^{\prime}, 5^{\prime}}\right), 7.34\left(\mathrm{dd},{ }^{3} J_{H H \text { tran }}=17.1 \mathrm{~Hz},{ }^{3} J_{H H \text { cis }}=10.7\right.$ 
$\left.\mathrm{Hz}, 1 \mathrm{H}, \mathrm{CH}=\mathrm{CH}_{2}\right), 7.27-7.22\left(\mathrm{~m}, 3 \mathrm{H}, \mathrm{Ph}^{2^{\prime}, 4^{\prime}, 6^{\prime}}\right), 5.69\left(\mathrm{dd},{ }^{3} J_{H H \text { trans }}=17.1 \mathrm{~Hz},{ }^{2} J_{H H}=1.5 \mathrm{~Hz}, 1 \mathrm{H}\right.$, $\left.\mathrm{CH}=\mathrm{CH}_{2}\right), 5.48\left(\mathrm{dd},{ }^{3} J_{H H}\right.$ cis $\left.=10.7 \mathrm{~Hz},{ }^{2} J_{H H}=1.5 \mathrm{~Hz}, 1 \mathrm{H}, \mathrm{CH}=\mathrm{CH}_{2}\right)$.

${ }^{13} \mathrm{C}\left\{{ }^{1} \mathrm{H}\right\}-\mathrm{NMR}\left(\delta, 20^{\circ} \mathrm{C}, \mathrm{CDCl}_{3}, 125 \mathrm{MHz}\right): 159.0(1 \mathrm{C}, C=\mathrm{N}), 152.6\left(1 \mathrm{C}^{\mathrm{q}}, \mathrm{Ph}^{1}\right), 139.3\left(1 \mathrm{C}^{\mathrm{q}}\right.$, $\left.\mathrm{Ph}^{2}\right), 133.9\left(1 \mathrm{C}, \mathrm{CH}=\mathrm{CH}_{2}\right), 133.2\left(1 \mathrm{C}^{\mathrm{q}}, \mathrm{Ph}^{1}\right), 131.2\left(1 \mathrm{C}, \mathrm{Ph}^{4}\right), 129.3,128.1,127.2\left(5 \mathrm{C}, \mathrm{Ph}^{3,3^{\prime}, 5,5^{\prime}, 6}\right)$, $126.1\left(1 \mathrm{C}, \mathrm{Ph}^{4}\right), 121.1\left(2 \mathrm{C}, \mathrm{Ph}^{2 \prime} 6^{\prime}\right), 118.7\left(1 \mathrm{C}, \mathrm{CH}=\mathrm{CH}_{2}\right)$.

Preparation of 1 for direct use in the synthesis of 4: A mixture of aniline (17 mg, $0.18 \mathrm{mmol})$ and $\mathrm{NEt}_{3}$

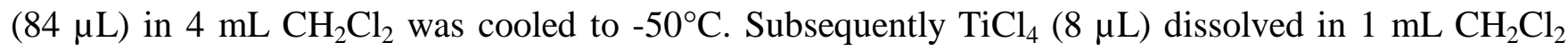
was added slowly under an inert atmosphere of argon followed by slow addition of 2-vinylbenzaldehyde (21 $\mathrm{mg}, 0.16 \mathrm{mmol})$. The cooling bath was removed and the reaction was stirred for $20 \mathrm{~h}$ at room temperature. After removal of the solvent the resulting residue was crushed with a spatula. After addition of $10 \mathrm{~mL} \mathrm{Et}_{2} \mathrm{O}$ the resulting suspension was stirred vigorously until the solid content was grounded to a fine powder. This powder was sucked off and evaporation of the ether released a yellow oil which was used without further purification. Yield: $30 \mathrm{mg}(94 \%)$. NMR analysis of the crude product revealed the presence of $\mathbf{1}$ and approx. 5\% of aniline.

\section{Synthesis of (SPY-5-31)-Dichloro- $\left(\kappa^{2}(C, N)-2-((E)-(\right.$ phenylimino $)$ methyl)benzylidene-(1,3-} bis(2,4,6-trimethylphenyl)4,5-dihydroimidazol-2-ylidene)-ruthenium (4)

A Schlenk flask was charged with 3 (42 mg, $0.0625 \mathrm{mmol})$ and a solution of crude 1 (20 mg, 0.097 mmol) in $4 \mathrm{~mL} \mathrm{CH} \mathrm{Cl}_{2}$ under inert atmosphere of argon. The reaction mixture was stirred at room temperature for 15 hours. Removal of the solvent in vacuum gave a green wax, which was dissolved in $1 \mathrm{~mL} \mathrm{CH} \mathrm{Cl}_{2}$. Upon addition of pentane/ $\mathrm{Et}_{2} \mathrm{O}$ a green precipitate formed which was filtered off and dried in vacuum. Yield: $34 \mathrm{mg}(89 \%)$.

Anal. Calcd for $\mathrm{C}_{35} \mathrm{H}_{37} \mathrm{Cl}_{2} \mathrm{~N}_{3} \mathrm{Ru}$ (MW: 671.66): C, 62.59; H, 5.55. Found: C, 62.45; H, 5.67.

${ }^{1} \mathrm{H}-\mathrm{NMR}\left(\delta, 20^{\circ} \mathrm{C}, \mathrm{CDCl}_{3}, 500 \mathrm{MHz}\right): 18.74(\mathrm{~s}, 1 \mathrm{H} \mathrm{Ru}=\mathrm{CH}), 8.65(\mathrm{~s}, 1 \mathrm{H}, \mathrm{CH}=\mathrm{N}), 7.69(\mathrm{t}, 1 \mathrm{H}$, $\mathrm{Ph}^{5}$ ), 7.51-7.46 (m, 2H, $\left.\mathrm{Ph}^{3,6}\right), 7.24\left(\mathrm{t}, 1 \mathrm{H}, \mathrm{Ph}^{4}\right), 7.15$ (t, 2H, $\left.\mathrm{Ph}^{3^{\prime}, 5^{\prime}}\right), 7.09$ (bs, 4H, $\left.\mathrm{Mes}^{3,5,3^{\prime}, 5^{\prime}}\right), 7.07$ (d, $\left.2 \mathrm{H}, \mathrm{Ph}^{2}, 6^{\prime}\right), 6.80\left(\mathrm{~d}, 1 \mathrm{H}, \mathrm{Ph}^{4}\right), 4.14\left(\mathrm{~m}, 4 \mathrm{H}, \mathrm{N}-\mathrm{CH}_{2}\right), 2.48,\left(\mathrm{bs}, 18 \mathrm{H}, \mathrm{CH}_{3}{ }^{\mathrm{Mes}}\right)$.

${ }^{13} \mathrm{C}\left\{{ }^{1} \mathrm{H}\right\}-\mathrm{NMR}\left(\delta, 20^{\circ} \mathrm{C}, \mathrm{CDCl}_{3}, 125 \mathrm{MHz}\right): 311.9(1 \mathrm{C}, \mathrm{Ru}=\mathrm{CH}), 214.7\left(1 \mathrm{C}^{\mathrm{q}}, \mathrm{NHC}\right), 167.6$ $(1 \mathrm{C}, \mathrm{C}=\mathrm{N}), 151.3\left(1 \mathrm{C}^{\mathrm{q}}, \mathrm{Ph}^{1}{ }^{\prime}\right), 142.8,138.7\left(2 \mathrm{C}^{\mathrm{q}}, \mathrm{Ph}^{1,2}\right), 135.6,133.9\left(3 \mathrm{C}, \mathrm{Mes}^{4,4^{\prime}}, \mathrm{Ph}^{4}\right), 129.8,129.0$ (6C, $\left.\mathrm{Mes}^{3,3^{\prime}, 5,5^{\prime}} \mathrm{Ph}^{3^{\prime}, 5^{\prime}}\right), 128.8,127.4,126.7\left(3 \mathrm{C}, \mathrm{Ph}^{4,5,6}\right), 122.8\left(1 \mathrm{C}, \mathrm{Ph}^{3}\right), 122.2\left(2 \mathrm{C}, \mathrm{Ph}^{2^{\prime}, 6^{\prime}}\right), 52.4$, 
51.2(bs, 2C, N-CH ), $21.5\left(2 \mathrm{C}, \mathrm{CH}_{3}{ }^{\text {Mes4,4' }}\right.$ ), 20.3, 18.6 (bs, $4 \mathrm{C}, \mathrm{CH}_{3}{ }^{\text {Mes2,2' } 6,6^{\prime}}$ ); (signals for Mes ${ }^{1,1^{\prime} 2,2^{\prime} 6,6^{\prime}}$ were not observed).

\section{Synthesis of $(E)$-N-benzylidene-2-vinylbenzenamine (2)}

A mixture of 2-vinylphenylamine (200 mg, $1.7 \mathrm{mmol})$ and $\mathrm{NEt}_{3}(0.93 \mathrm{~mL})$ in $20 \mathrm{~mL} \mathrm{CH}_{2} \mathrm{Cl}_{2}$ was cooled to $-50^{\circ} \mathrm{C}$. Subsequently $\mathrm{TiCl}_{4}(90 \mu \mathrm{L})$ dissolved in $2 \mathrm{~mL} \mathrm{CH}_{2} \mathrm{Cl}_{2}$ was added slowly under an inert atmosphere of argon followed by slow addition of benzaldehyde (200 mg, $1.9 \mathrm{mmol})$. The cooling bath was removed and the reaction stirred for 20 hours at room temperature. After removal of the solvent the resulting residue was crushed with a spatula. After addition of $10 \mathrm{~mL} \mathrm{Et}_{2} \mathrm{O}$ the resulting suspension was stirred vigorously until the solid content was grounded to a fine powder. This powder was sucked off and evaporation of the ether released a yellow oil, which solidified upon addition of pentane. The product was recrystallized from pentane. Yield: $48 \mathrm{mg}(13 \%)$.

Anal. Calcd for $\mathrm{C}_{15} \mathrm{H}_{13} \mathrm{~N}$ (MW: 207.27): C, 86.92; H, 6.32. Found: C, 86.79; H, 6.39.

${ }^{1} \mathrm{H}-\mathrm{NMR}\left(\delta, 20^{\circ} \mathrm{C}, \mathrm{CDCl}_{3}, 500 \mathrm{MHz}\right): 8.38(\mathrm{~s}, 1 \mathrm{H}, \mathrm{N}=\mathrm{CH}), 7.93\left(\mathrm{~m}, 2 \mathrm{H}, \mathrm{Ph}^{2,6^{\prime}}\right), 7.59\left(\mathrm{dd},{ }^{3} J_{H H}\right.$ $\left.=7.8 \mathrm{~Hz},{ }^{4} \mathrm{~J}_{\mathrm{HH}}=1.5 \mathrm{~Hz}, 1 \mathrm{H}, \mathrm{Ph}^{3}\right), 7.57-7.48\left(\mathrm{~m}, 3 \mathrm{H}, \mathrm{Ph}^{3,} 4^{4}, 5^{\prime}\right), 7.31-7.18\left(\mathrm{~m}, 3 \mathrm{H}, \mathrm{Ph}^{4,5}, \mathrm{CH}=\mathrm{CH}_{2}\right)$, $6.97\left(\mathrm{~d},{ }^{3} J_{H H}=7.0 \mathrm{~Hz}, 1 \mathrm{H}, \mathrm{Ph}^{6}\right), 5.75\left(\mathrm{dd},{ }^{3} J_{H H}\right.$ trans $\left.=18.1 \mathrm{~Hz},{ }^{2} J_{H H}=1.5 \mathrm{~Hz}, 1 \mathrm{H}, \mathrm{CH}=\mathrm{CH}_{2}\right), 5.29(\mathrm{dd}$, ${ }^{3} J_{H H c i s}=11.2 \mathrm{~Hz},{ }^{2} J_{H H}=1.5 \mathrm{~Hz}, 1 \mathrm{H}, \mathrm{CH}=\mathrm{CH}_{2}$ ).

${ }^{13} \mathrm{C}\left\{{ }^{1} \mathrm{H}\right\}$-NMR $\left(\delta, 20^{\circ} \mathrm{C}, \mathrm{CDCl}_{3}, 125 \mathrm{MHz}\right): 160.3(1 \mathrm{C}, C=\mathrm{N}), 150.0\left(1 \mathrm{C}, \mathrm{Ph}^{1}\right), 136.4(1 \mathrm{C}$, $\left.\mathrm{Ph}^{1^{\prime}}\right), 133.5\left(1 \mathrm{C}, \mathrm{CH}=\mathrm{CH}_{2}\right), 131.6\left(1 \mathrm{C}, \mathrm{Ph}^{4}\right), 131.4\left(1 \mathrm{C}, \mathrm{Ph}^{2}\right), 129.0,128.9\left(4 \mathrm{C}, \mathrm{Ph}^{2^{\prime}, 3^{\prime}, 5^{\prime}, 6^{\prime}}\right), 128.8$, $126.0,125.8\left(3 \mathrm{C}, \mathrm{Ph}^{3,4,5}\right), 118.6\left(1 \mathrm{C}, \mathrm{Ph}^{6}\right), 114,9\left(1 \mathrm{C}, \mathrm{CH}=\mathrm{CH}_{2}\right)$.

Preparation of $\mathbf{2}$ for direct use in the synthesis of 5: A mixture of 2-vinylphenylamine (20 $\mathrm{mg}, 0.17$ $\mathrm{mmol})$ and $\mathrm{NEt}_{3}(93 \mu \mathrm{L})$ in $4 \mathrm{~mL} \mathrm{CH}_{2} \mathrm{Cl}_{2}$ was cooled to $-50^{\circ} \mathrm{C}$. Subsequently $\mathrm{TiCl}_{4}(9 \mu \mathrm{L}$, ) dissolved in $1 \mathrm{~mL} \mathrm{CH}_{2} \mathrm{Cl}_{2}$ was added slowly under an inert atmosphere of argon followed by slow addition of benzaldehyde ( $20 \mathrm{mg}, 0.17 \mathrm{mmol}$ ). The cooling bath was removed and the reaction stirred for 20 hours at room temperature. After removal of the solvent the resulting residue was crushed with a spatula. After addition of $10 \mathrm{~mL} \mathrm{Et}_{2} \mathrm{O}$ the resulting suspension was stirred vigorously until the solid content was grounded to a fine powder. This powder was sucked off and evaporation of the ether released a yellow oil which was used without further purification. Yield: $32 \mathrm{mg}$ (93\%). NMR analysis of the crude product revealed the presence of $\mathbf{1}$ and approx. 7\% of benzaldehyde. 
Synthesis of (SPY-5-31)-Dichloro- $\left(\kappa^{2}(C, N)-(E)-2-(b e n z y l i d e n e a m i n o) b e n z y l i d e n e-(1,3-b i s(2,4,6-\right.$ trimethylphenyl)4,5-dihydroimidazol-2-ylidene)-ruthenium (5)

5 was prepared analogously to 4 using $3(45 \mathrm{mg}, 0.0619 \mathrm{mmol})$ and crude 2 (19 $\mathrm{mg}, 0.0917$ mmol) as the starting materials. Yield: $36 \mathrm{mg}(86 \%)$.

Anal. Calcd for $\mathrm{C}_{35} \mathrm{H}_{37} \mathrm{Cl}_{2} \mathrm{~N}_{3} \mathrm{Ru}$ (MW: 671.66): C, 62.59; H, 5.55. Found: C, 62.55; H, 5.78.

${ }^{1} \mathrm{H}-\mathrm{NMR}\left(\delta, 20^{\circ} \mathrm{C}, \mathrm{CDCl}_{3}, 500 \mathrm{MHz}\right): 17.07(\mathrm{~s}, 1 \mathrm{H} \mathrm{Ru}=\mathrm{CH}), 9.32(\mathrm{~s}, 1 \mathrm{H}, \mathrm{CH}=\mathrm{N}), 7.90(\mathrm{~d}, 2 \mathrm{H}$, $\left.\mathrm{Ph}^{2^{\prime}, 6^{\prime}}\right), 7.51-7.44\left(\mathrm{~m}, 2 \mathrm{H}, \mathrm{Ph}^{4,5}\right), 7.35\left(\mathrm{~d}, 1 \mathrm{H}, \mathrm{Ph}^{6}\right), 7.19\left(\mathrm{t}, 2 \mathrm{H}, \mathrm{Ph}^{3{ }^{\prime}, 5^{\prime}}\right), 7.13\left(\mathrm{t}, 1 \mathrm{H}, \mathrm{Ph}^{4}\right), 7.08(\mathrm{~s}, 4 \mathrm{H}$, $\left.\mathrm{Mes}^{3,3^{\prime}, 5,5^{\prime}}\right), 6.86\left(\mathrm{~d}, 1 \mathrm{H}, \mathrm{Ph}^{3}\right), 4.05\left(\mathrm{~s}, 4 \mathrm{H}, \mathrm{N}-\mathrm{CH}_{2}\right), 2.49$ (bs, 12H, $\left.\mathrm{CH}_{3}{ }^{\mathrm{Mes} 2,2^{\prime} 6,6^{\prime}}\right), 2.46(\mathrm{~s}, 6 \mathrm{H}$, $\left.\mathrm{CH}_{3}{ }^{\mathrm{Mes} 4,4^{\prime}}\right)$.

${ }^{13} \mathrm{C}\left\{{ }^{1} \mathrm{H}\right\}-\mathrm{NMR}\left(\delta, 20^{\circ} \mathrm{C}, \mathrm{CDCl}_{3}, 125 \mathrm{MHz}\right): 260.4$ (b, 1C, $\left.\mathrm{Ru}=C \mathrm{H}\right), 212.0(1 \mathrm{C}, \mathrm{NHC}), 164.8$ $(1 \mathrm{C}, \mathrm{CH}=\mathrm{N}), 154.7\left(1 \mathrm{C}, \mathrm{Ph}^{1}\right), 144.3\left(1 \mathrm{C}, \mathrm{Ph}^{1}\right), 138.6,138.5\left(6 \mathrm{C}, \mathrm{Mes}^{1,1^{\prime}, 2,2^{\prime}, 6,6^{\prime}}\right), 134.8,131.6,130.2$, 128.2 (6C, $\left.\mathrm{Mes}^{4,4^{\prime}} \mathrm{Ph}^{2,4,4^{\prime}, 5}\right), 129.9,129.4$ (8C, $\left.\mathrm{Mes}^{3,3^{\prime}, 5,5^{\prime}} \mathrm{Ph}^{2^{\prime}, 3^{\prime}, 5^{\prime}, 6^{\prime}}\right), 120.5,117.0\left(2 \mathrm{C}, \mathrm{Ph}^{3,6}\right), 52.1(2 \mathrm{C}$, $\left.\mathrm{N}-\mathrm{CH}_{2}\right), 21.4\left(2 \mathrm{C}, \mathrm{CH}_{3}{ }^{\text {Mes4,4'}}\right), 19.3$ (bs, $4 \mathrm{C}, \mathrm{CH}_{3}{ }^{\text {Mes 2,2',6,6' }}$ ). 


\section{Crystal Structure Determination}

Crystals of the complexes $\mathbf{4}$ and $\mathbf{5}$ were obtained by layering saturated solutions in $\mathrm{CH}_{2} \mathrm{Cl}_{2}$ with $\mathrm{Et}_{2} \mathrm{O}$ and subsequent diffusion at room temperature. $\mathbf{4}$ crystallized as unsolvated green plates, $\mathbf{5}$ formed solvated green prisms of $\mathbf{5} \cdot \mathrm{solv}$ with disordered solvent molecules (believed to be predominantly $\mathrm{Et}_{2} \mathrm{O}$ because of lacking high solvent electron densities indicative for presence of significant amounts of $\mathrm{CH}_{2} \mathrm{Cl}_{2}$ ). X-ray data were collected on a Bruker AXS Smart CCD area detector diffractometer at room temperature using graphite monochromatized Mo-K $\alpha$ radiation, $\lambda=0.71073 \AA$, and $0.3^{\circ} \omega-$ scan frames covering complete spheres of the reciprocal space. After data integration with program SAINT, corrections for absorption, $\lambda / 2$ effect of the detector, and crystal decay were applied to the data using program SADABS. The structures were solved by direct methods using program SHELXS97. Structure refinement on $F^{2}$ was carried out with program SHELXL97. All non-hydrogen atoms were refined anisotropically. Hydrogen atoms were inserted in idealized positions and were refined riding with the atoms to which they were bonded. In case of $\mathbf{4}$ an orientation disorder of the rutheniumdichloro 2-[(E)phenyliminomethyl]-benzylidene moiety was found, which corresponded to a $\sim 180^{\circ}$ rotation about the $\mathrm{Ru}-\mathrm{C}_{\text {imidazole }}$ bond for about $25 \%$ of the complexes (space filling properties of this moiety similar for both orientations). This disorder was taken into account by refinement with geometric restraints using instruction SAME of program SHELXL97. In case of 5.solv, the disordered solvent was taken into account by four carbon atom positions $\mathrm{C}(1 \mathrm{~s})$ though $\mathrm{C}(4 \mathrm{~s})$, the coordinates and population parameters of which were allowed to vary. Salient crystallographic data are compiled in Table S1 (compound 4) and Table S2 (compound 5.solv). Atomic coordinates, thermal displacement parameters, bond lengths, and bond angles of $\mathbf{4}$ and $\mathbf{5} \cdot$ solv are given as a CIF (see Supporting Information).

Following programs were used in the calculations:

Bruker, programs SMART, version 5.054; SAINT, version 6.2.9; SADABS version 2.10; XPREP, version 5.1; SHELXTL, version 5.1. Bruker AXS Inc., Madison, WI, USA, 2001.

Sheldrick, G. M., SHELX97: Program System for Crystal Structure Determination, University of Göttingen, Germany, 1997. 
Table S1. Crystal data and structure refinement for compound 4.

\begin{tabular}{|c|c|}
\hline Identification code & $931 \mathrm{cmfr} 2$ \\
\hline Empirical formula & C35 H37 Cl2 N3 Ru \\
\hline Formula weight & 671.65 \\
\hline Temperature & $297(2) \mathrm{K}$ \\
\hline Wavelength & $0.71073 \AA$ \\
\hline Crystal system, space group & Monoclinic, $\mathrm{C} 2 / \mathrm{c}$ \\
\hline Unit cell dimensions & $\begin{array}{ll}\mathrm{a}=38.806(2) \AA & \alpha=90 \mathrm{deg} . \\
\mathrm{b}=10.9768(6) \AA & \beta=103.486(1) \mathrm{deg} . \\
\mathrm{c}=15.7674(8) \AA & \gamma=90 \mathrm{deg} .\end{array}$ \\
\hline Volume & $6531.1(6) \AA^{3}$ \\
\hline Z, Calculated density & $8, \quad 1.366 \mathrm{Mg} / \mathrm{m}^{3}$ \\
\hline Absorption coefficient & $0.671 \mathrm{~mm}^{-1}$ \\
\hline$F(000)$ & 2768 \\
\hline Crystal size & $0.65 \times 0.50 \times 0.03 \mathrm{~mm}$, green plate \\
\hline Diffractometer & $\begin{array}{l}\text { Bruker SMART APEX CCD platform type 3-circle } \\
\text { (sealed X-ray tube, Mo K } \alpha \text { radiation, graphite } \\
\text { monochr., CCD at } 50 \mathrm{~mm} \text { in } 512 \times 512 \text { pixel mode) }\end{array}$ \\
\hline Scan type / width / speed & $\begin{array}{l}\omega \text {-scan frames / } \Delta \omega=0.3^{\circ} / 20 \text { sec. per frame } \\
\text { full sphere data collection, } 4 \times 600 \text { frames }\end{array}$ \\
\hline Theta range for data collection & 2.16 to $30.01 \mathrm{deg}$ \\
\hline Index ranges & $-54<=\mathrm{h}<=54,-15<=\mathrm{k}<=15,-22<=1<=22$ \\
\hline Reflections collected / unique & $47727 / 9398[R($ int $)=0.0285]$ \\
\hline Completeness to theta $=30.01$ & $98.6 \%$ \\
\hline Absorption correction & Multi-scan (program SADABS) \\
\hline Max. and min. transmission & 1.00 and 0.89 \\
\hline Structure solution & Direct methods (program SHELXS97) \\
\hline Refinement method & Full-matrix least-squares on $F^{2}$ (prg SHELXL97) \\
\hline Data / restraints / parameters & $9398 / 0 / 377$ \\
\hline Goodness-of-fit on $\mathrm{F}^{2}$ & 1.046 \\
\hline Final R indices [I>2sigma(I)] & $\mathrm{R} 1=0.0498, \mathrm{wR} 2=0.1207$ \\
\hline $\mathrm{R}$ indices (all data) & $\mathrm{R} 1=0.0742, \mathrm{wR} 2=0.1342$ \\
\hline Largest diff. peak and hole & 0.97 and $-0.44 \mathrm{e}^{-3}$ \\
\hline
\end{tabular}

$\mathrm{R} 1=\Sigma|| \mathrm{F}_{\mathrm{o}}|-| \mathrm{F}_{\mathrm{c}} \| / \Sigma\left|\mathrm{F}_{\mathrm{o}}\right|, \quad \mathrm{wR} 2=\left[\Sigma\left(\mathrm{w}\left(\mathrm{F}_{\mathrm{o}}{ }^{2}-\mathrm{F}_{\mathrm{C}}{ }^{2}\right)^{2}\right) / \Sigma\left(\mathrm{w}\left(\mathrm{F}_{\mathrm{o}}{ }^{2}\right)^{2}\right)\right]^{3 / 2}$ 
Table S2. Crystal data and structure refinement for $\mathbf{5} \cdot$ solv.

\begin{tabular}{|c|c|}
\hline Identification code & $957 \mathrm{csfr}$ \\
\hline Empirical formula & $\mathrm{C} 35 \mathrm{H} 37 \mathrm{Cl} 2 \mathrm{~N} 3 \mathrm{Ru}$ \\
\hline Formula weight & 671.65 \\
\hline Temperature & $296(2) \mathrm{K}$ \\
\hline Wavelength & $0.71073 \AA$ \\
\hline Crystal system, space group & Monoclinic, $\mathrm{C} 2 / \mathrm{c}$ \\
\hline Unit cell dimensions & $\begin{aligned} \mathrm{a}=27.76(4) \AA & \alpha=90 \mathrm{deg} . \\
\mathrm{b}=15.49(2) \AA & \beta=92.59(4) \mathrm{deg} . \\
\mathrm{c}=17.149(5) \AA & \gamma=90 \mathrm{deg} .\end{aligned}$ \\
\hline Volume & $7366(15) \AA^{3}$ \\
\hline Z, Calculated density & $8, \quad 1.211 \mathrm{Mg} / \mathrm{m}^{3}$ \\
\hline Absorption coefficient & $0.595 \mathrm{~mm}^{-1}$ \\
\hline$F(000)$ & 2768 \\
\hline Crystal size & $0.75 \times 0.70 \times 0.65 \mathrm{~mm}$, green block \\
\hline Diffractometer & $\begin{array}{l}\text { Bruker SMART APEX CCD platform type 3-circle } \\
\text { (sealed X-ray tube, Mo Ko radiation, graphite } \\
\text { monochr., CCD at } 50 \mathrm{~mm} \text { in } 512 \times 512 \text { pixel mode) }\end{array}$ \\
\hline Scan type / width / speed & $\begin{array}{l}\omega \text {-scan frames } / \Delta \omega=0.3^{\circ} / 10 \mathrm{sec} \text {. per frame } \\
\text { full sphere data collection, } 4 \times 600 \text { frames }\end{array}$ \\
\hline Theta range for data collection & 2.38 to $25.00 \mathrm{deg}$ \\
\hline Index ranges & $-32<=\mathrm{h}<=32, \quad-18<=\mathrm{k}<=18, \quad-20<=1<=20$ \\
\hline Reflections collected / unique & $20346 / 6369[R($ int $)=0.0596]$ \\
\hline Completeness to theta $=25.00$ & $98.0 \%$ \\
\hline Absorption correction & Multi-scan (program SADABS) \\
\hline Max. and min. transmission & 1.00 and 0.86 \\
\hline Structure solution & Direct methods (program SHELXS97) \\
\hline Refinement method & Full-matrix least-squares on $\mathrm{F}^{2}$ (prg SHELXL97) \\
\hline Data / restraints / parameters & $6369 / 0 / 401$ \\
\hline Goodness-of-fit on $\mathrm{F}^{2}$ & 1.176 \\
\hline Final $R$ indices [I>2sigma(I)] & $\mathrm{R} 1=0.0586, \mathrm{wR} 2=0.1214$ \\
\hline $\mathrm{R}$ indices (all data) & $\mathrm{R} 1=0.0996, \mathrm{wR} 2=0.1692$ \\
\hline Largest diff. peak and hole & 1.50 and $-0.56{\mathrm{e} \AA^{-3}}^{-3.03}$ \\
\hline
\end{tabular}

$\mathrm{R} 1=\Sigma|| \mathrm{F}_{\mathrm{o}}|-| \mathrm{F}_{\mathrm{c}} \| / \Sigma\left|\mathrm{F}_{\mathrm{o}}\right|, \quad \mathrm{wR} 2=\left[\Sigma\left(\mathrm{w}\left(\mathrm{F}_{\mathrm{o}}{ }^{2}-\mathrm{F}_{\mathrm{C}}{ }^{2}\right)^{2}\right) / \Sigma\left(\mathrm{w}\left(\mathrm{F}_{\mathrm{o}}{ }^{2}\right)^{2}\right)\right]^{3 / 2}$

(§) Chemical formula and derived quantities are given without solvent. 


\section{Polymerization procedures}

Polymerization procedure in NMR tubes. Complexes 4 or 5 ( $1 \mathrm{mg}$; 1.0 equiv.) were weighed into the NMR tube and dissolved $\left(\mathrm{CDCl}_{3}, 0,5 \mathrm{~mL}\right.$ ). Monomer 6 (50 equiv.) was injected. ${ }^{1} \mathrm{H}-\mathrm{NMR}$ spectra were recorded every 3-4 days. The propagation was followed by integration of the olefinic proton resonances of the monomer and the polymer in dependence of time. Carbene peaks of the propagating species could not be detected.

Polymerization procedure in bulk. Complexes 4 or 5 ( $1 \mathrm{mg}$; 1.0 equiv.) were dissolved in $50 \mu \mathrm{L}$ of $\mathrm{CH}_{2} \mathrm{Cl}_{2}$ and added to monomer 6 (300 equiv.) placed in a Schlenk-tube. The reaction vessel was put into a preheated $\left(110^{\circ} \mathrm{C}\right)$ oil-bath for $15 \mathrm{~min}$. Almost immediately a solidification of the reaction mixture occurred. After cooling the reaction mixture to room-temperature, the residue was dissolved in $\mathrm{CH}_{2} \mathrm{Cl}_{2}$ and $50 \mu \mathrm{L}$ of ethylvinylether was added. Absence of 6 was confirmed by TLC. Afterwards, the solution was added slowly to vigorously stirred methanol. The resulting white precipitate was sampled and dried in vacuum. Yields ranged from $92-97 \%$.

Polymerization procedure in solution. Complexes 4 or 5 ( $1 \mathrm{mg}$; 1.0 equiv.) were dissolved in $3 \mathrm{~mL}$ toluene and added to monomer 6 (300 equiv.) placed in a Schlenk-tube. The reaction vessel was put into a preheated $\left(110^{\circ} \mathrm{C}\right)$ oil-bath for $15 \mathrm{~min}$. After cooling the reaction mixture to room-temperature, $50 \mu \mathrm{L}$ of ethylvinylether was added. Absence of $\mathbf{6}$ was confirmed by TLC. Afterwards, the solution was added slowly to vigorously stirred methanol. The resulting white precipitate was sampled and dried in vacuum. Yields ranged from $91-95 \%$.

Polymerization procedure in DSC pans. Defined solutions of 4 or $5(0.002 \mathrm{mmol} / \mathrm{mL}, 1.0$ equiv.) and monomer 6 (1.000 mmol/mL, 500 equiv.) in $\mathrm{CH}_{2} \mathrm{Cl}_{2}$ were prepared. Equal amounts of initiator and monomer were mixed on a watch glass and the solvent was evaporated at room temperature within 5-7 minutes. The resulting mixture was transferred into a DSC pan. The polymerisation was then followed by monitoring the heat-flow when employing a heating rate of $3^{\circ} \mathrm{C} / \mathrm{min}$.

\footnotetext{
${ }^{1}$ Kirmse, W.; Mrotzeck U.; Siegfried, R. Chem. Ber. 1991, 124, 241.

${ }^{2}$ Slugovc, C.; Perner, B.; Stelzer, F. Mereiter, K. Organometallics 2004, 23, 3622.

${ }^{3}$ Kerins, F.; O’Shea, D. F.; J. Org. Chem. 2002, 67, 4968.
} 